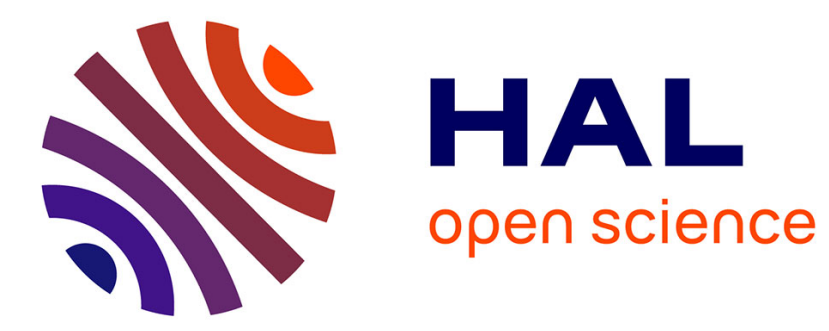

\title{
Combinatorial Surrogate-Assisted Optimization for Bus Stops Spacing Problem
}

Florian Leprêtre, Cyril Fonlupt, Sébastien Verel, Virginie Marion

\section{To cite this version:}

Florian Leprêtre, Cyril Fonlupt, Sébastien Verel, Virginie Marion. Combinatorial Surrogate-Assisted Optimization for Bus Stops Spacing Problem. Biennial International Conference on Artificial Evolution (EA 2019), Oct 2019, Mulhouse, France. hal-02319558

\section{HAL Id: hal-02319558 \\ https://hal.science/hal-02319558}

Submitted on 18 Oct 2019

HAL is a multi-disciplinary open access archive for the deposit and dissemination of scientific research documents, whether they are published or not. The documents may come from teaching and research institutions in France or abroad, or from public or private research centers.
L'archive ouverte pluridisciplinaire HAL, est destinée au dépôt et à la diffusion de documents scientifiques de niveau recherche, publiés ou non, émanant des établissements d'enseignement et de recherche français ou étrangers, des laboratoires publics ou privés. 


\title{
Combinatorial Surrogate-Assisted Optimization for Bus Stops Spacing Problem
}

\author{
Florian Leprêtre ${ }^{1}$, Cyril Fonlupt ${ }^{1}$, Sébastien Verel $^{1}$, and Virginie Marion ${ }^{1}$ \\ Univ. Littoral Côte d'Opale, LISIC \\ Calais, France, F-62100 \\ florian.lepretre@univ-littoral.fr
}

\begin{abstract}
The distribution of transit stations constitutes an ubiquitous task in large urban areas. In particular, bus stops spacing is a crucial factor that directly affects transit ridership travel time. Hence, planners often rely on traffic surveys and virtual simulations of urban journeys to design sustainable public transport routes. However, the combinatorial structure of the search space in addition to the time-consuming and black-box traffic simulations require computationally expensive efforts. This imposes serious constraints on the number of potential configurations to be explored. Recently, powerful techniques from discrete optimization and machine learning showed convincing to overcome these limitations. In this preliminary work, we build combinatorial surrogate models to approximate the costly traffic simulations. These so-trained surrogates are embedded in an optimization framework. More specifically, this article is the first to make use of a fresh surrogate-assisted optimization algorithm based on the mathematical foundations of discrete Walsh functions in order to solve the real-world bus stops spacing optimization problem. We conduct our experiments with the SIALAC benchmark in the city of Calais, France. We compare state-of-the-art approaches and we highlight the accuracy and the optimization efficiency of the proposed methods.
\end{abstract}

Keywords: bus stops spacing $\cdot$ combinatorial optimization $\cdot$ surrogate models

\section{Motivations}

The United Nations expect sixty percent of the world's population to live in urban areas by the next decade [2]. This relentlessly growing rate constantly challenges urban planners to design sustainable cities so as to improve the mobility of their inhabitants and travellers. This objective can be achieved, in a way, by an efficient planning and management of public transport systems, such as trams, buses or even self-service bicycles. The correct design of these systems is the key to offer potential users a competitive transit mode compared to the private car. This is particularly advocated for mitigating environmental impacts of transport and could also help to revitalize and renew interest in some districts of the city. For example, the location of a transit station in an area where none 
currently exists may attract new customers to public transport who previously lacked the service.

Substantial works exist in the litterature on the deployment of such efficient transport systems. A recurring challenge is to ensure that transit stops are properly spaced. Without loss of generality, the present article focuses on the optimization of bus stops spacing. It is well known that their distribution represents an important factor directly affecting passengers travel times [21,27]. Some research even investigates social costs, economic benefits or environmental impacts of bus stations positionings [24,19]. Also, several methods are introduced to find optimal spacings for minimal travel costs. On the one hand, coverage models have been widely employed, such as the Thiesen polygons [27] or the Voronoi diagrams [28]. These techniques allow additional data to be assessed while searching for optimal stop positions (e.g., district density around the stop). On the other hand, the discretization of the study areas has also shown promising. Ibeas et. al. proposed to split the transit route area in small links of equal distance [11]. Each link represents a potential stop location, and the so-discretized optimization problem is solved with a pattern-search iterative algorithm [9]. Besides, Furth et. al. considered each intersection of the studied road network as a potential stop location [8]. Then, a dynamic programming algorithm was used to determine the optimal bus stop positions.

Inspired by the aforementioned works, this article considers the bus stops spacing problem as a pseudo-boolean problem, where the passenger travel time is the fitness function to minimize. Then, a binary variable is associated with each possible location for a bus stop: the variable equals one if the stop is activated or equals zero if not - this will be discussed further in section 3. However, such studies as well as many others in the literature mostly rely on numerical simulations of urban traffic flows, which are usually blackbox models. As a consequence, only the design variables and the resulting values of the simulation are known. Moreover, it is often computationally time expensive (from minutes to hours) to get the fitness value of one single simulation $[3,6]$. In addition with the combinatorial explosion of the search space, optimization experts thus face a serious limitation on the capacity to freely explore potential solutions. To tackle such an optimization challenge, one classical solution in Surrogate-assisted Optimization $(\mathrm{SaO})$ is to learn a surrogate model to approximate the costly simulator evaluations and then reduce the number of potential sampled solutions during the search process. Although the field of combinatorial surrogate models has long received little attention, it is now experiencing a sudden renewed interest, bringing with it new algorithmic ideas to the community $[5,4,22]$.

As part of this preparatory and applicative work for further studies, we implement a variety of recent combinatorial surrogate modeling techniques to approximate the time-expensive traffic simulations. Further, we embed the so-learnt surrogates in the context of bus stops spacing optimization. In particular, this work is the first to make use of a newly published $\mathrm{SaO}$ algorithm based on the mathematical foundations of discrete Walsh functions coupled with powerful grey-box optimization techniques [14], in order to solve a class of real-world 
problems. We aim to highlight the accuracy and the optimization performances of these methods.

The rest of this paper is organized as follows. In section 2, we introduce the state-of-the-art combinatorial surrogate modeling techniques and the foundations of $\mathrm{SaO}$. Section 3 is devoted to experiments specifications and their analyses. In section 4 , we conclude the paper and discuss future works.

\section{Combinatorial Surrogate-Assisted Optimization}

Surrogate models formulate quick-to-evaluate mathematical models, so as to approximate black-box and time-consuming computations. They are built from a sample of evaluated solutions. Therefore, the main purpose of surrogate-assisted optimization is to efficiently select the solutions to be sampled in order to quickly improve the quality of the surrogate and thus the quality of the solutions found.

A surrogate-assisted method combines three components (see Algorithm 1). The first component is the surrogate model itself which is a regression model of the fitness function. The model must be expressive enough to catch the complexity of the fitness function, but at the same time slightly sophisticated in order to ease the learning when a small sample of solutions is available. The second component is an acquisition function defined from the surrogate model. This acquisition function can be directly the surrogate model or a trade-off between the predicted quality of candidate solution and the estimation error of the surrogate model. The goal is to guide the search and to ensure a balance between exploration that increases the quality of the surrogate model and exploitation that pushes towards high-quality solutions according to the surrogate model. The last component is the algorithm to optimize the acquisition function. This algorithm has to be efficient in time and in quality to converge quickly to the promising solutions given by the acquisition function. Therefore, such promising solution is selected, evaluated and added to the sample for the next iteration of the search algorithm. An efficient surrogate-assisted optimizer for combinatorial problems is a relevant combination of these three components.

To the best of our knowledge, four main methods have been proposed for pseudo-boolean problems: Radial Basis Function model [16], Kriging approach [26], Bayesian approach [4] and Walsh basis functions decomposition [22].

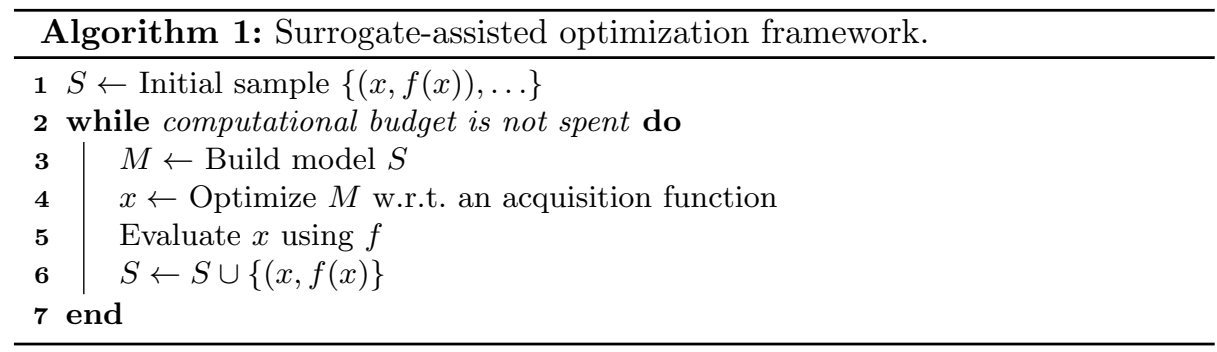


Kriging approach Kriging approach is a direct extension of the numerical surrogate approach and is based on Gaussian Process (GP) [26,25]. In the context of combinatorial structures, Euclidean distance is replaced by the Hamming distance for pseudo-boolean functions or more sophisticated discrete distance for the search space of permutations. Then, Kriging is coupled with the Efficient Global Optimizer framework (EGO) [12]. This framework takes advantage of the uncertainty of the approximations given by the GP and uses a genetic algorithm to select the promising solution that maximizes the Expected Improvement (EI), i.e., the acquisition function. One should note that the computational complexity of EI is high and can not be reduced by some classic techniques in combinatorial optimization such as incremental evaluation. Nevertheless, this surrogate-assisted optimization has been shown to outperform the aforementioned Radial Basis Function model [16] - thus the latter will not be detailed here.

Bayesian approach Another state-of-the-art surrogate-assisted approach is the Bayesian Optimization of Combinatorial Structures (BOCS) algorithm [4]. The statistical model of BOCS is the standard multilinear polynomial of binary variables. Therefore, Baptista et. al. argue that the model takes into account the interactions between the binary variables. Only a quadratic polynomial model is studied in their article (i.e., one variable interacts with only other one):

$$
\forall x \in\{0,1\}^{n}, M_{2}(x)=a_{0}+\sum_{i \in N} a_{i} x_{i}+\sum_{i<j \in N} a_{i j} x_{i} x_{j},
$$

where $N=\{1, \ldots, n\}$. The regression technique is the Sparse Bayesian Linear Regression [15]. The optimizer is a basic simulated annealing that minimizes the approximation of the fitness function provided by the surrogate model with a regularization term.

Walsh basis functions A new combinatorial surrogate model based on Walsh functions has been proposed [22]. Walsh functions [23] describe a normal and orthogonal basis of discontinuous functions that can be employed to decompose any function of the Hilbert space. Therefore, Verel et. al. assumed that the expensive pseudo-boolean functions might be substituted by a polynomial of Walsh decompositions of order $k$ :

$$
\forall x \in\{0,1\}^{n}, W_{k}(x)=\sum_{\ell \text { s.t. } o(\ell) \leqslant k} w_{\ell} \cdot(-1)^{\sum_{i=1}^{n} \ell_{i} x_{i}},
$$

where $o$ is the order of the Walsh function, i.e., the number of binary digits equals to 1 in the binary representation of $\ell$. In the following, we restrain to quadratic interactions:

$$
\forall x \in\{0,1\}^{n}, W_{2}(x)=w_{0}+\sum_{i=1}^{n} w_{i}(-1)^{x_{i}}+\sum_{i<j \in N} w_{i j}(-1)^{x_{i}+x_{j}} .
$$


To face the quadratic number of polynomial terms, the regression technique is a linear model trained with $\ell_{1}$-norm as regularizer, aka the Lasso [20]. Recently, the Walsh Surrogate-assisted Optimization (WSaO) algorithm has been introduced [14]. The authors benefit from powerful grey-box optimization techniques and use the so-called Efficient Hill-climber (EH) from Chicano et. al. [7] as an optimizer for the Walsh surrogates. WSaO has been shown to outperform both Kriging and BOCS approaches, scaling up at least to dimension $n=100$.

\section{Experiments}

\subsection{Overview}

We consider the bus stops spacing problem as a pseudo-boolean optimization challenge. For the sake of simplicity in this preliminary work, the focus is on one regular bus line in the city of Calais, France.

Bus stops All potential bus stops are implemented in advance on the given road network. They are manually located on intersection nodes and are numbered from 1 to $n$, where $n$ is the total number of potential stops in the bus route, following [8]. Then, a solution to the optimization problem, i.e., a possible design of the bus stops, is denoted by a binary string $x \in\{0,1\}^{n}$. Therefore, open bus stops are associated to bits in $x$ equal to one, whereas closed stops are associated to bits equal to zero. The first and last stops are constrained to be open. Only open bus stops are taken into account during the forthcoming simulations. Figure 1 illustrates a simplified bus stops design on the studied bus route. The complete bus route considers $n=20$ potential stop locations.

Urban flows The simulation system considered in this work is the Multi Agent Transport Simulation (MATSim) [10]. MATSim requires as inputs a road network model [18] and the initial mobility scenarios for a set of agents (i.e., a set of travelers' schedules). These scenarios are generated according to the SIALAC Benchmark [13]. The latter allows to synthesize mobility plans which assess such information as living quarters, business districts or main entry and exit points of the city. As an example, Figure 2 illustrates a scenario where agents are distributed into four living quarters. The present work studies six scenarios involving 5000 travelers with different number of home and working area clusters. The possible home cluster number is 1 cluster, 4 clusters or uniform when the population is randomly distributed over the city area; they are denoted as $1 h, 4 h$ or $u h$, respectively. The possible working area cluster number is 1 or 4 clusters; they are denoted as $1 a$ or $4 a$, respectively. Finally, a scenario defines a round trip between a home location and an activity location, for each traveler. The latter are distributed according to the configuration of the six studied scenarios: $1 h-1 a, 1 h-4 a, 4 h-1 a, 4 h-4 a, u h-1 a$, and $u h-4 a$. The number of cluster impacts the travel time for pedestrians and cars which allows to test the robustness of $\mathrm{SaO}$ algorithms. One simulation with MATSim, i.e., one fitness function evaluation, requires about a minute of computation as a single-thread program. 


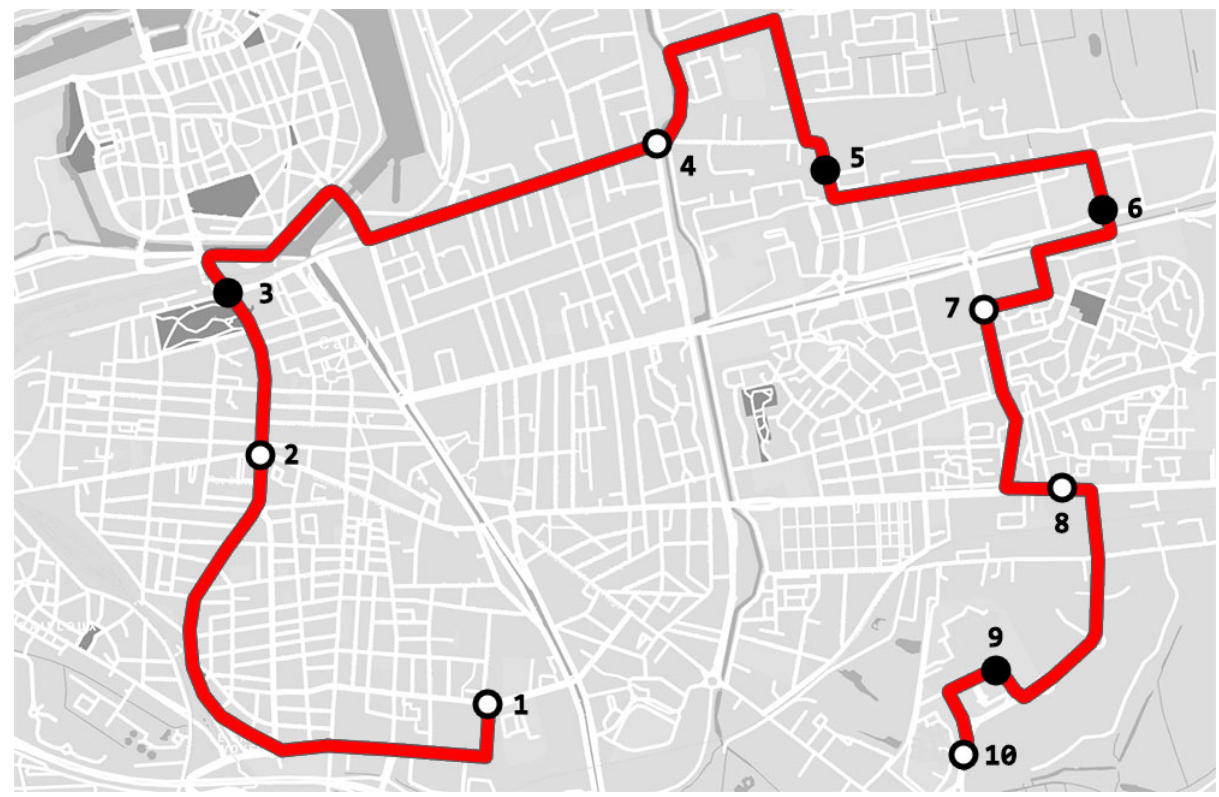

Figure 1. Simplified example of open or closed bus stops (white or black dots) on the regular bus route (red line), according to the solution $x=1101001101$.
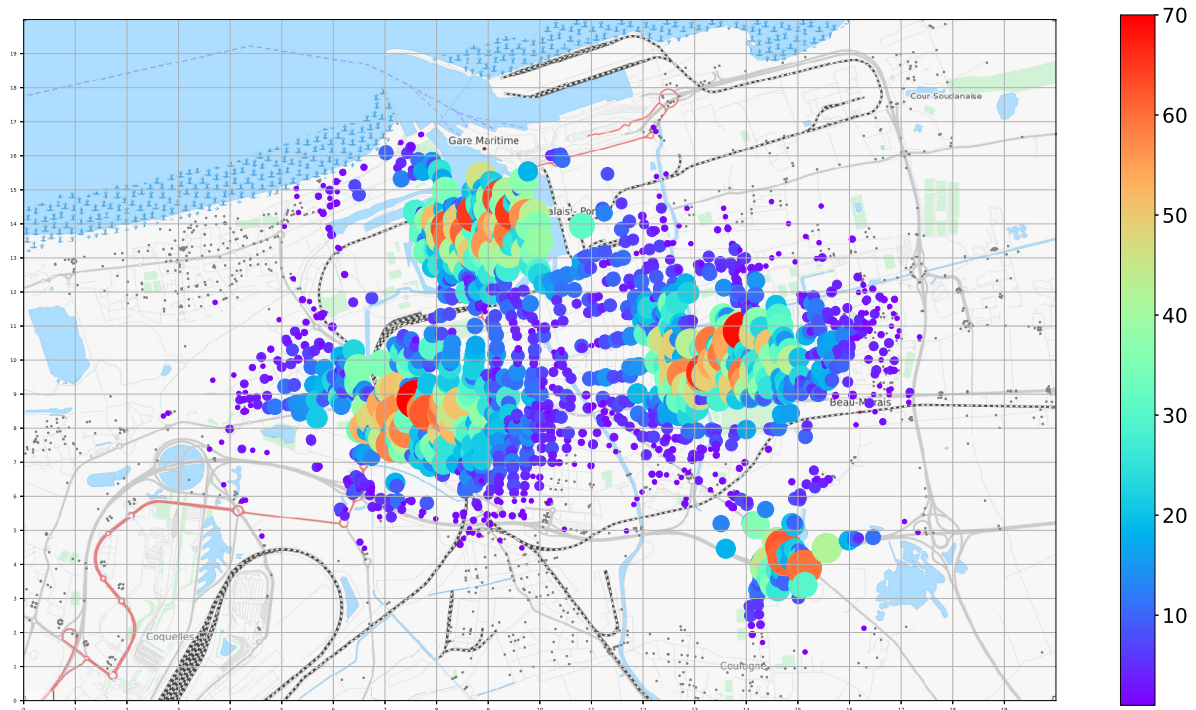

Figure 2. Four home clusters inside Calais road network. Colors indicate the number of agents departing from a node. 
Experimental setup We aim to minimize the travelers mean travel time, i.e., the fitness function computed by MATSim, i.e., the black-box simulator. Then, we use binary strings of dimension $n=20$, corresponding to the 20 potential bus stops locations. We follow the experimental setup exposed in [14], except that we validate the accuracy of the models against a test-set of 250 solutions generated uniformly at random and we restrain to quadratic interactions for BOCS and $\mathrm{WSaO}$ methods. Algorithms and experiments are fully implemented in Python, using standard machine learning and optimization packages [17].

\subsection{Accuracy of Surrogate Models}

We first benchmark the accuracy of three surrogate models based on gaussian process (Kriging), multilinear polynomials used in BOCS, and Walsh polynomials. No optimization algorithm is involved yet. Selected solutions to learn the black-box simulator are sampled randomly from $\{0,1\}^{n}$. Figure 3 compares the mean absolute error made by the models as a function of the random sample size dedicated to their learning. Although Kriging seems promising in the very first iterations, the computational effort required for a slight improvement in accuracy increases considerably as the learning process progesses. For both Walsh, and multilinear based methods, the convergence of the model quality is reached around a sample size of 400 solutions on 3 scenarios (1h-1a, 4h-1a and uh-1a). For more difficult scenarios $1 \mathrm{~h}-4 \mathrm{a}, 4 \mathrm{~h}-4 \mathrm{a}$ and $\mathrm{uh}-4 \mathrm{a}$, the precision quality increases beyond the largest sample size. Overall scenarios, Walsh surrogates appear as the most accurate models. On all scenario, the precision gain of Walsh vs. multilinear polynomial is approximatively $30 \%$ for the largest sample size of $10^{3}$.

\subsection{Performances of Optimizers}

We compare state-of-the-art $\mathrm{SaO}$ algorithms presented in section 2. In addition, we also compare the performances of multilinear polynomials embedded with an Iterated Local Search based on the Efficient Hill-climber (EH) [7]. Unlike the regression analysis of the previous section and according to Algorithm 1, the solution added each iteration to the surrogate's learning sample is now the solution that minimizes the surrogate model. Figure 4 plots the minimization of the fitness as a function of the learning sample, for one SIALAC scenario. At a glance, EGO algorithm stalls as soon as the sample size gets bigger than 100. This unsuitable scaling-up was already identified in [14]. However, WSaO seems promising with a small learning sample, while a multilinear polynomial coupled with BOCS appears better when the sample size grows (see Table 1). However, notice that the order of difference between the two approaches is only in seconds of mean travel time. Both methods seems promising for that moderate size scenario with one bus line, and 20 potential bus stop positions. These results are still under active work and follow the first results performed on artificial benchmarks [14]. 

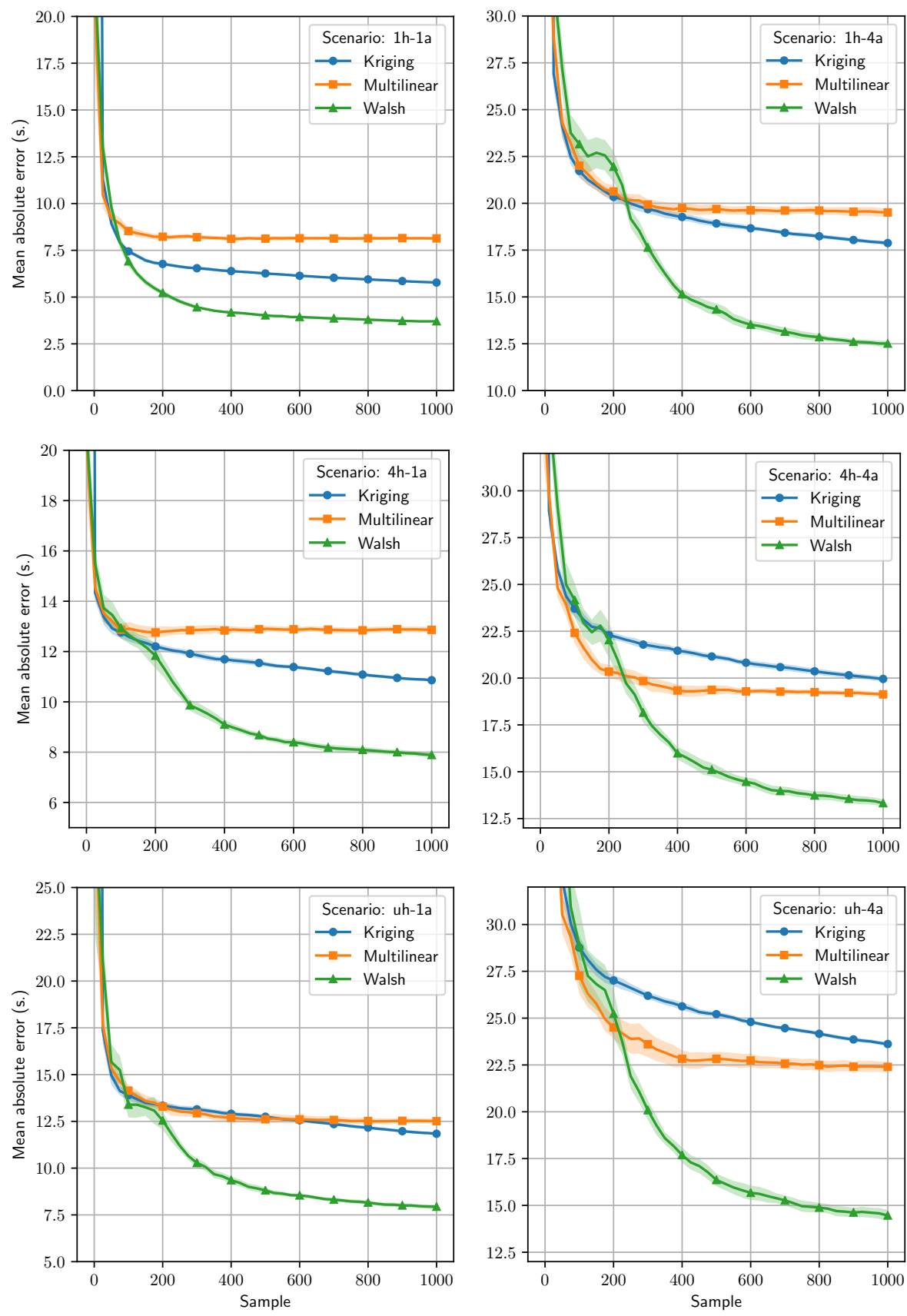

Figure 3. Mean absolute error with confidence interval as a function of random samples for six SIALAC scenarios ( $h$ : home clusters, $a$ : activity clusters). The lower the better. 


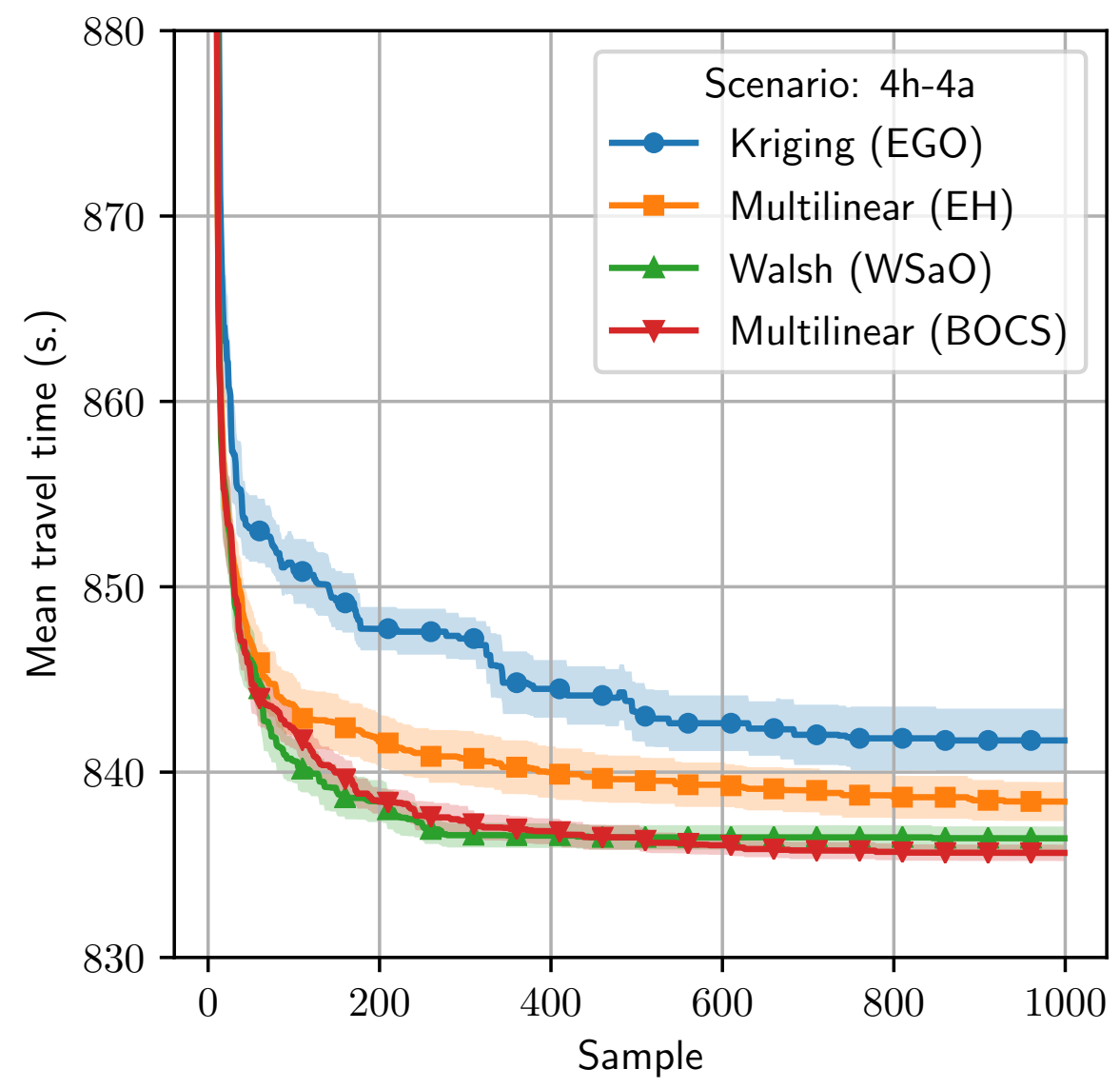

Figure 4. Minimization of mean travel times according to sample size for one SIALAC scenario. The lower the better.

Table 1. Average mean travel times according to the learning sample size. The lower the better. Values appear in bold when they are statistically significant with MannWhitney tests at level $5 \%$.

\begin{tabular}{|c||cccc|}
\hline Sample size & Kriging (EGO) & Multilinear (EH) & Walsh (WSaO) & Multilinear (BOCS) \\
\hline 100 & $850.82 \pm 4.00$ & $843.55 \pm 4.17$ & $\mathbf{8 4 0 . 5 9} \pm \mathbf{3 . 0 3}$ & $842.24 \pm 2.58$ \\
400 & $844.49 \pm 3.55$ & $839.99 \pm 3.56$ & $\mathbf{8 3 6 . 5 7} \pm \mathbf{1 . 7 0}$ & $\mathbf{8 3 6 . 8 1} \pm \mathbf{1 . 7 8}$ \\
1000 & $841.71 \pm 3.27$ & $838.41 \pm 2.68$ & $836.43 \pm 1.62$ & $\mathbf{8 3 5 . 6 4} \pm \mathbf{1 . 1 3}$ \\
\hline
\end{tabular}




\section{Discussion}

Combinatorial surrogate models succeed to learn the time-consuming and blackbox traffic simulator, with a reasonable error lower than three percent of the average real simulator responses. The results show that polynomial-based models coupled with grey-box optimization algorithms are competitive against standard state-of-the-art methods in a surrogate-assisted optimization purpose. EGO approach quickly stalls, while $\mathrm{WSaO}$ and BOCS approaches converge to satisfying bus route designs. In particular, this article is the first to apply WSaO to solve such a class of real-world optimization problem.

This preparatory work opens many directions for future applicative research. First, we would like to scale up the bus stop spacing problem to a hundred dimensions at least, in order to design more concise bus routes and to get more challenging problems to face for state-of-the-art $\mathrm{SaO}$ methods. As it was pointed out in [14], a higher dimension would allow to draw more clearly conclusions as to the most appropriate polynomial decomposition. Further, these polynomial models are restrained here to quadratic interactions between variables. The cubic, or higher interaction is envisaged in order to aspire to the conception of more accurate models. Finally in a more applicative way, we are considering to redraw a part of the bus routes, based on the results obtained from $\mathrm{SaO}$ algorithms. Such routes could be implemented in the Zenbus [1] vizualisation platform, which could represent a powerful tool for urban planners and decisionmakers.

\section{Acknowledgments}

Experiments presented in this paper were carried out using the CALCULCO computing platform, supported by SCOSI / ULCO (Service COmmun du Système d'Information de l'Université du Littoral Côte d'Opale). We are grateful to PMCO for its funding, and we thank Calais city (France) for the data and its support.

\section{References}

1. Zenbus. https://zenbus.net/sitac-calais, accessed: 2019-05-31

2. The world's cities in 2016. United Nations, Department of Economic and Social Affairs (2016)

3. Armas, R., Aguirre, H., Zapotecas-Martínez, S., Tanaka, K.: Traffic signal optimization: minimizing travel time and fuel consumption. In: International Conference on Artificial Evolution (Evolution Artificielle). pp. 29-43. Springer (2015)

4. Baptista, R., Poloczek, M.: Bayesian optimization of combinatorial structures. In: International Conference on Machine Learning (ICML). pp. 462-471 (2018)

5. Bartz-Beielstein, T., Zaefferer, M.: Model-based methods for continuous and discrete global optimization. Applied Soft Computing 55, 154-167 (2017)

6. Branke, J.: Simulation optimization tutorial. In: Proceedings of the Genetic and Evolutionary Computation Conference Compagnion. ACM (2018) 
7. Chicano, F., Whitley, D., Sutton, A.M.: Efficient identification of improving moves in a ball for pseudo-boolean problems. In: Proceedings of the 2014 Annual Conference on Genetic and Evolutionary Computation. pp. 437-444. GECCO '14, ACM, New York, NY, USA (2014)

8. Furth, P., B Rahbee, A.: Optimal bus stop spacing through dynamic programming and geographic modeling. Transportation Research Record 1731, 15-22 (2000)

9. Hooke, R., Jeeves, T.A.: " direct search" solution of numerical and statistical problems. J. ACM 8(2), 212-229 (Apr 1961)

10. Horni, A., Nagel, K., Axhausen, K. (eds.): Multi-Agent Transport Simulation MATSim. Ubiquity Press, London (Aug 2016)

11. Ibeas, Á., dell'Olio, L., Alonso, B., Sainz, O.: Optimizing bus stop spacing in urban areas. Transportation Research Part E: Logistics and Transportation Review 46(3), $446-458(2010)$

12. Jones, D.R., Schonlau, M., Welch, W.J.: Efficient global optimization of expensive black-box functions. Journal of Global optimization 13(4), 455-492 (1998)

13. Leprêtre, F., Fonlupt, C., Verel, S., Marion, V.: Sialac benchmark: on the design of adaptive algorithms for traffic lights problems. In: Proceedings of the Genetic and Evolutionary Computation Conference Companion. pp. 288-289. ACM (2018)

14. Leprêtre, F., Fonlupt, C., Verel, S., Marion, V.: Walsh functions as surrogate model for pseudo-boolean optimization problems. In: Proceedings of the Genetic and Evolutionary Computation Conference. ACM (2019)

15. Makalic, E., Schmidt, D.F.: A simple sampler for the horseshoe estimator. IEEE Signal Processing Letters 23(1), 179-182 (2016)

16. Moraglio, A., Kattan, A.: Geometric generalisation of surrogate model based optimisation to combinatorial spaces. In: European Conference on Evolutionary Computation in Combinatorial Optimization. pp. 142-154. Springer (2011)

17. Pedregosa, F., Varoquaux, G., Gramfort, A., Michel, V., Thirion, B., Grisel, O., Blondel, M., Prettenhofer, P., Weiss, R., Dubourg, V., Vanderplas, J., Passos, A., Cournapeau, D., Brucher, M., Perrot, M., Duchesnay, E.: Scikit-learn: Machine learning in Python. Journal of Machine Learning Research 12, 2825-2830 (2011)

18. Ramm, F., Karch, C., Topf, J.: Geofabrik. https://www.geofabrik.de, accessed: 2018-01-24

19. Saka, A.A.: Model for determining optimum bus-stop spacingin urban areas. Journal of Transportation Engineering 127(3), 195-199 (2001)

20. Tibshirani, R., Wainwright, M., Hastie, T.: Statistical learning with sparsity: the lasso and generalizations. Chapman and Hall/CRC (2015)

21. Vaughan, R., Cousins, E.: Optimum location of stops on a bus route. In: International Symposium on Transportation and Traffic Theory, 7th, 1977, Kyoto, Japan (1977)

22. Verel, S., Derbel, B., Liefooghe, A., Aguirre, H., Tanaka, K.: A surrogate model based on walsh decomposition for pseudo-boolean functions. In: International Conference on Parallel Problem Solving from Nature. pp. 181-193. Springer (2018)

23. Walsh, J.L.: A closed set of normal orthogonal functions. American Journal of Mathematics 45(1), 5-24 (1923)

24. Wirasinghe, S.C., Ghoneim, N.S.: Spacing of bus-stops for many to many travel demand. Transportation Science 15(3), 210-221 (1981)

25. Zaefferer, M., Stork, J., Bartz-Beielstein, T.: Distance measures for permutations in combinatorial efficient global optimization. In: International Conference on Parallel Problem Solving from Nature. pp. 373-383. Springer (2014)

26. Zaefferer, M., Stork, J., Friese, M., Fischbach, A., Naujoks, B., Bartz-Beielstein, T.: Efficient global optimization for combinatorial problems. In: GECCO (2014) 
27. Zheng, C., Zheng, S., Ma, G.: The bus station spacing optimization based on game theory. Advances in Mechanical Engineering 7(2), 453979 (2015)

28. Zhu, Z., Guo, X., Chen, H., Zeng, J., Wu, J.: Optimization of urban mini-bus stop spacing: A case study of shanghai (china). Tehnicki Vjesnik 24, 949-955 (06 2017) 